\title{
The region of Fe II emission line formation of the symbiotic nova RR Telescopii ${ }^{\star}$
}

\author{
D. Kotnik-Karuza ${ }^{1}$, M. Friedjung ${ }^{2}$, and P. L. Selvelli ${ }^{3}$ \\ 1 Physics Department, University of Rijeka, Omladinska 14, HR 51000 Rijeka, Croatia \\ 2 Institut d'Astrophysique, Paris, France \\ e-mail: friedjung@iap.fr \\ 3 Osservatorio Astronomico, Trieste, Italy \\ e-mail: selvelli@ts.astro.it
}

Received 12 April 2001 / Accepted 15 October 2001

\begin{abstract}
In our investigation of the region of formation of the Fe II emission lines in RR Tel, we have applied the SAC method to the optical Fe II emission line fluxes measured by Crawford et al. (1999). It is possible to determine physical parameters of the line emitting region, because of the presence both of lines with large and much smaller optical thicknesses. The values we obtained, given in Tables 3 and 4 , are limits in most cases. The upper limit to the excitation temperature of the metastable levels in the permitted line region of $6600 \mathrm{~K}$, is close to the value of this temperature in the forbidden line region. Other excitation temperature limits corresponding to level population ratios were also found. The permitted lines are formed in a region with a radius between $1.8 \times 10^{12} \mathrm{~cm}$ and $1.6 \times 10^{14} \mathrm{~cm}$, while the forbidden lines are formed in a region with a minimum radius of $5.3 \times 10^{12} \mathrm{~cm}$. The populations of the lower levels of the permitted lines indicate a minimum column density of $\mathrm{Fe}+$ of $3.0 \times 10^{18}$ atoms $\mathrm{cm}^{-2}$ and, by assuming cosmic abundances, to a minimum column density of $7.5 \times$ $10^{22}$ atoms $\mathrm{cm}^{-2}$ for $\mathrm{H}$. If we assume a minimum radius of $1.0 \times 10^{14} \mathrm{~cm}$, which is supposed to be that of dust condensation of the cool Mira component of this symbiotic binary, a minimum mass loss rate of $3.5 \times 10^{-6} M_{\mathrm{o}} \mathrm{yr}^{-1}$ is found. Our results support a model according to which both the permitted and the forbidden lines are formed in the wind of the cool Mira component above the regions where absorption by dust is important. The forbidden and the permitted lines are not formed in quite the same region, the former being most probably formed further out in the part of an accelerated wind which is ionized by the hot component.
\end{abstract}

Key words. stars: binaries: symbiotic - stars: individual: RR Tel - stars: fundamental parameters

\section{Introduction}

RR Tel belongs to the group of symbiotic novae that are interacting binaries consisting of a late-type giant and a hot companion thought to be a white dwarf. They are distinguished from classic symbiotic stars because only one single major outburst, with a recovery phase typically lasting for decades, has been observed.

Since its last outburst in 1944, RR Tel has been the subject of many extensive spectroscopic investigations concerning its nature, which indicate a very complex physical situation, involving a white dwarf, a Mira variable of

Send offprint requests to: D. Kotnik-Karuza, e-mail: kotnik@mapef.pefri.hr

* Tables 1 and 2 are only available in electronic form at the CDS via anonymous ftp to cdsarc.u-strasbg.fr (130.79.128.5) or via

http://cdsweb.u-strasbg.fr/cgi-bin/qcat?J/A+A/381/507 spectral type near M5 and pulsation period of 387 days, planetary nebula-like plasma and additional hot plasma that could be due to colliding winds from the two binary components (Contini \& Formiggini 1999). The evidence for a circumstellar dust shell leads to a classification of RR Tel as a D-type symbiotic (Feast \& Glass 1974).

The slowly evolving optical spectrum consists almost entirely of emission lines, while the spectrum has progressed to higher degrees of excitation with increasing time. Thus, in addition to being a fascinating and complex astronomical object, RR Tel is recognized as an excellent source of emission lines that may be used to provide insights into atomic data not readily obtainable by other means (Thackeray 1977; Penston et al. 1983; McKenna et al. 1997).

Numerous permitted and forbidden Fe II emission lines occur in a large spectral range, from the UV to the IR. They have great potential as a means of determining 


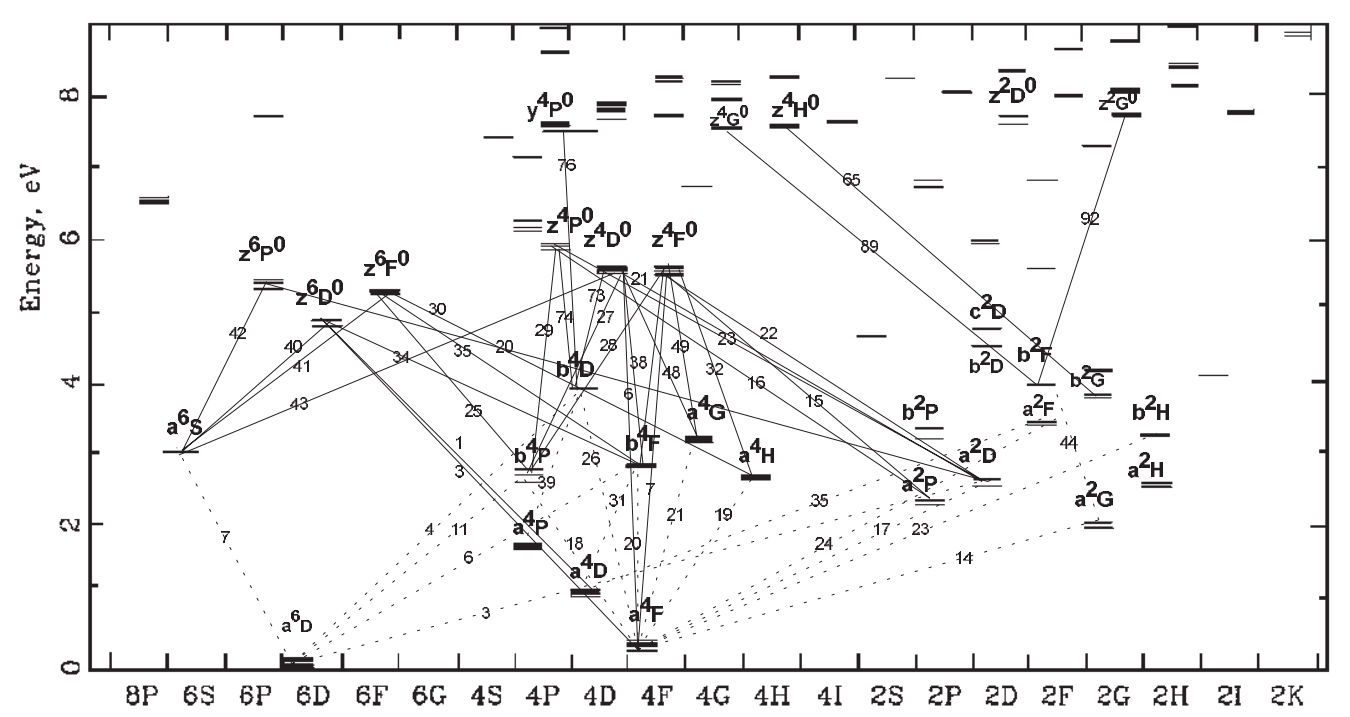

Fig. 1. Energy distribution of the lowest levels of Fe II and multiplets observed in the 3180-9455 $\AA$ emission spectrum of RR Tel. The horizontal axis shows terms ranged from octets to doublets. The vertical scale indicates energy of levels in eV. For each multiplicity and orbital momentum, even terms are on the left and odd terms are on the right. Permitted and forbidden transitions are marked with solid and dotted lines respectively. The associated numbers refer to different multiplets.

the physical conditions in the emitting regions, provided the necessary atomic data are available (Muratorio \& Friedjung 1988). A partial Grotrian diagram of $\mathrm{Fe}^{+}$ is shown in Fig. 1. Let it be noted that Hayes and Nussbaumer (Hayes \& Nussbaumer 1986) found physical conditions using other lines.

In this work we have studied RR Tel by the Self Absorption Curve (SAC) method (Friedjung \& Muratorio 1980; Friedjung \& Muratorio 1987; Muratorio \& Friedjung 1988; Baratta et al. 1998) extensively applied in spectroscopic studies of emitting stellar regions since 1987 . The approach is different than that of Viotti et al. (2001), where RR Tel is discussed together with other astronomical objects. Here we obtain information about the size of the line emitting region, its column density and the mass loss rate of the Mira variable.

\section{Observations and methods of analysis}

We have used the flux calibrated high resolution spectrum of RR Tel observed by Crawford et al. (Crawford et al. 1999), which covers the wavelength range from $3180 \AA$ to $9455 \AA$. The high spectral resolution of $6 \mathrm{~km} \mathrm{~s}^{-1}$ made reliable intensity and FWHM measurement possible also for very weak and sharp features respectively.

The database of Crawford et al. (Crawford et al. 1999) was checked critically regarding not only the line identifications but also the line intensities, at least for the lines and spectral ranges decisive for the physical parameters and their limits, determined in this work.

The list of Fe II identifications has been checked exploiting the high resolution of a UVES spectrum of RR Tel (Selvelli \& Bonifacio 2000), in which the intrinsic sharpness of the Fe II emissions against other emission lines constitutes an unambiguous criterion.
The flux calibration was tested by comparing our dataset with another published optical spectrum of RR Tel (McKenna et al. 1997), taking into account the fact that the Fe II line intensities did not vary much over the 3-year period between the observations (Keenan 2001).

Since the measured lines in (McKenna et al. 1997) cover the 3430-9320 $\AA$ range, the comparison with the spectrum used by us (Crawford et al. 1999) could be made only in the overlapping 3430-9320 $\AA$ region. The calibrated fluxes agree in the limits of the declared errors bellow $6000 \AA$. The forbidden red lines at larger wavelengths, do not deviate very much.

Outside the 3490-5530 ^ range Crawford et al. (1999) made calibrations using the expected relative intensities of He II lines, so the corrections to the measured fluxes in that range could not be those for relative interstellar absorption. The explanation of what was done is unfortunately missing from the paper. One of the authors of it, F. P. Keenan, reassured us however; according to him the relative line intensities should be good at least to the $20 \%$ level for lines not widely separated in wavelength (Keenan 2001). The effect of the uncertainty in the nature of the corrections in the red, of the order of 0.1 dex, on our results is not large.

The rapid variation of the measured/corrected fluxes given by Crawford et al. over the small wavelength range below $3400 \AA$ made it however necessary to treat the published results for that range with some caution. This rapid variation was due to poor observing conditions, which influenced the standard star flux (Keenan 2001). According to him, comparing lines widely separated in wavelength like the red and near UV could lead to errors of $30 \%$. The possible influence of the less accurate intensities in the near UV wavelength region has been taken into account in this paper. 
The atomic data were taken from different sources (Kurucz 1981; Garstang 1962; Hansen \& Lister 1988; Nussbaumer et al. 1981; Moore 1945; Muratorio 1999).

The accepted permitted (Table 1) and forbidden lines (Table 2) are separately available in electronic form at $\mathrm{CDS}$.

A total number of 131 lines belonging to different multiplets originating from transitions between the lowest levels with an excitation potential up to $9 \mathrm{eV}$ (Fig. 1) have been used in applying the Friedjung-Muratorio self absorption curve (SAC) method (Friedjung \& Muratorio 1987). The method has proved to be effective in determining limits to relative populations of upper and lower terms, as well as in deriving other information about the line formation region.

The method has not been applied exactly as in previous papers, so we summarize it and its assumptions briefly.

The observational log-log plot of the quantity $F \lambda^{3} / g f$ derived from the line flux $F$ emitted in a transition versus $(g f \lambda)$, which is related to the optical thickness of that transition, is used to find the Self Absorption Curve (SAC) whose shape is determined by self absorption effects (Muratorio et al. 1992). Simple theory gives

$\log \frac{F \lambda^{3}}{g f}=\log \left(\frac{2 \pi^{2} e^{2} h R^{2}}{m_{\mathrm{e}} d^{2}}\right)+\log \left(\frac{N_{\mathrm{u}}}{g_{\mathrm{u}}}\right)+Q\left(\tau_{0}\right)$

and

$\log \tau_{0}=\log (g f \lambda)+\log \frac{N_{\mathrm{l}}}{g_{\mathrm{l}}}+\log \frac{\pi e^{2}}{m_{\mathrm{e}} c}-\log v_{\mathrm{d}}$

We call the left hand side of Eq. (1) the logarithm of the "normalized flux".

In Eqs. (1) and (2) $F$ is the emission line flux, $R$ the size of the emitting region perpendicular to the line of sight, $d$ the distance of the object, $N_{\mathrm{u}}$ the upper level column density, $N_{\mathrm{l}}$ the lower level column density, and $g_{\mathrm{u}}$, $g_{1}$ their statistical weights. $v_{\mathrm{d}}$ is the characteristic width of the line in velocity units.

$Q\left(\tau_{0}\right)$ is the SAC function, discussed by Kastner (Kastner 1999) for the case of plane parallel ("slab") geometry, the simplest geometry possible.

As long as level populations inside a spectroscopic term are proportional to their statistical weights, the log normalized flux against $\log (g f \lambda)$ values for each multiplet lie on a curve. For different multiplets the corresponding curves have relative vertical displacements because of different populations of the upper term and relative horizontal displacements due to differing populations of the lower term.

A type of excitation temperature of the line emitting region can be defined by

$\frac{\frac{N_{\mathrm{u}}}{g_{\mathrm{u}}}}{\frac{N_{1}}{g_{\mathrm{l}}}}=\mathrm{e}^{-\frac{\left(E_{\mathrm{u}}-E_{1}\right)}{k T}}=\mathrm{e}^{-\frac{1.44}{\lambda T}}$

The possibilities of estimating it are limited by observational uncertainties in the exact shape of the log normalized flux against $\log (g f \lambda)$ curves.
The SAC function will have always the same shape when population ratios between different line upper levels and also those between different line lower levels do not vary (including LTE when the excitation temperature is constant). The assumption of shape constancy is still approximately valid if most emission comes from regions where these population ratios do not change much, but is questionable if emission is received from different regions where the ratios of population densities of levels are not the same.

An upper level excitation temperature, given by $T=$ $5040 \cdot \Delta E_{21}(\mathrm{eV}) / \Delta Y$, can be obtained by use of the vertical shift $\Delta Y=\log \frac{\frac{N_{\mathrm{u} 1}}{g_{\mathrm{u} 1}}}{\frac{N_{\mathrm{u} 2}}{g_{\mathrm{u} 2}}}$ between empirical log normalized flux against $\log (g f \lambda)$ plots for two multiplets with a common lower term, such empirical plots being shown in Fig. 2 for several multiplets having the same lower term.

Similarly the lower level excitation temperature, of multiplets having the same upper term, $T=5040$. $\Delta E_{21}(\mathrm{eV}) / \Delta X$, the horizontal shift $\Delta X$ being equal to $\log \frac{\frac{N_{11}}{g_{11}}}{\frac{N_{12}}{g_{12}}}$, can be determined if the lines are fairly saturated.

Plots of $\log$ normalized flux against $\log (g f \lambda)$ for multiplets having the same upper term are shown in Fig. 3.

Another kind of upper to lower level population ratio can be also obtained by comparing the emission of a permitted line with that of a forbidden line, whose upper term is the lower term of a permitted line. This ratio, obtained from Eq. (1), can also be expressed as a temperature by (3). That temperature and corresponding population ratio, obtained from the normalized flux of the optically thin permitted line plateau, should be lower limits for the permitted line region, as forbidden lines do not need to be formed in the same region as permitted lines.

It was in addition possible to obtain a metastable level excitation temperature for all forbidden lines, assuming that they are optically thin. The value $\log \left(F \lambda^{3} / g f\right)$ is anticorrelated with the excitation potential, because as we shall see, these level populations follow a Boltzmann distribution, the temperature being found from the slope of the graph.

When $Q\left(\tau_{0}\right)$ becomes the function for the uniform slab of Friedjung \& Muratorio (1987), the size of the region where radiation of a particular line at a wavelength $\lambda$ is emitted, or more precisely its radius $R$, is given by

$R^{2}=\frac{F \lambda^{4} d^{2}}{2 \pi h c v_{\mathrm{d}}\left(1-\mathrm{e}^{-\tau_{0}}\right) \cdot \mathrm{e}^{-\frac{1.44}{\lambda T}}}$

We can simply understand what we are doing, if we bear in mind that when we determine the surface area perpendicular to the line of sight and so $R$, we are in fact dividing the total number of emitting atoms by the column density.

We assume the uniform slab self absorption curve as an approximation, because we do not know the true shape very well. Such radii will be correctable at a future date. In fact the lines are formed almost certainly in an inhomogeneous region. A best obtainable characteristic radius 


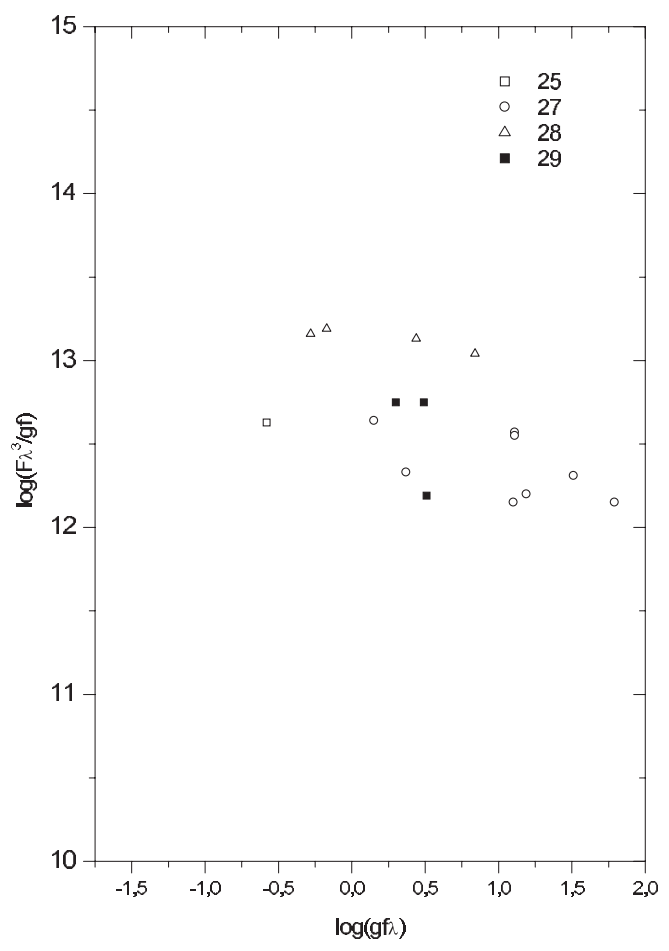

(a)

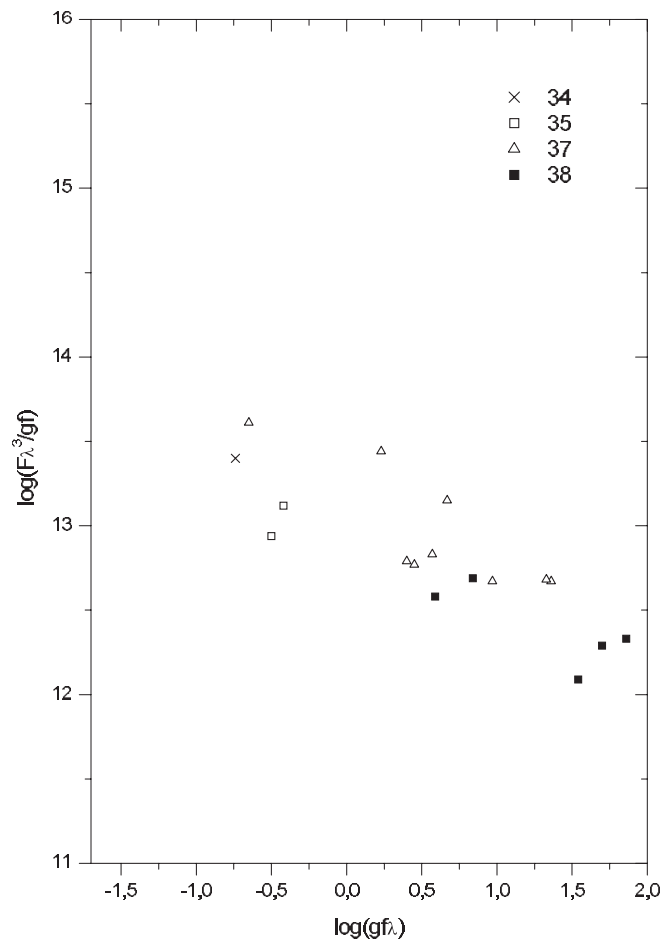

(b)

Fig. 2. Self absorption curves of multiplets with a common lower term a) $b^{4} \mathrm{P}$-multiplets: $25,27,28,29 ;$ b) b ${ }^{4} \mathrm{~F}-$ multiplets: $34,35,37,38$.

is that of rather weak lines, with not too large optical depths, which are near the plateau of the SAC. Strong lines are not typical of the whole line formation region. The assumption $\tau_{0}=1$ will in fact eventually lead to only a limit value of the radius.

The forbidden line region radius can also be determined, assuming that the excitation temperature given by the approximate Boltzmann distribution of their upper term populations, is the same as that between the upper and lower terms. In such a case only minimum radii can be found since the forbidden lines are usually optically thin.

As we see later we can also obtain limits on the column density from metastable level excitation temperatures.

\section{Results and discussion}

\subsection{NLTE effects}

The question of whether the populations are really in LTE can be considered both theoretically and empirically. From a theoretical point of view the populations are in LTE for the lowest levels when both excitation and de-excitation are dominated by collisions, that is at electron densities of more than $10^{7} \mathrm{~cm}^{-3}$ (Nussbaumer \& Storey 1988). The general problem of the approach to LTE was also discussed by Verner et al. (Verner et al. 1999) for emitting material with a high iron abundance. Their calculations show that Fe II tends to LTE at the limits of high particle and radiation densities, as well as at high Lyman continuum optical depths. The metastable levels numbered 10 and 25 tend to approach LTE at electron densities of more than $10^{8} \mathrm{~cm}^{-3}$ with an electron temperature of $T_{\mathrm{e}}=5000 \mathrm{~K}$. When the gas density $n_{\mathrm{H}}$ is $10^{9} \mathrm{~cm}^{-3}, T_{\mathrm{e}}=8000 \mathrm{~K}$ and the incident radiation has a Planckian energy distribution with a colour temperature also of $8000 \mathrm{~K}$, all the metastable levels, but not the higher levels, are in LTE for any radiation density. Finally even high level populations reach LTE for a Lyman continuum optical depth of more than 10 at the Lyman limit, when $T_{\mathrm{e}}=15000 \mathrm{~K}, n_{\mathrm{H}}=10^{9} \mathrm{~cm}^{-3}$ and at very low ionizing radiation density.

We can also look for non-LTE effects empirically. In particular there remains the question of the extent of possible NLTE effects of levels inside the same upper term of the observed permitted and forbidden transitions. In our test, which has been carried out with the assumption that the line lower levels (metastable for the permitted lines and near $0 \mathrm{eV}$ for the forbidden lines) are in LTE, we checked for NLTE effects inside each term by comparing the lines with upper levels having different $J$ 's. They neither show systematic deviations as a function of $J$ for the permitted nor for the forbidden transitions, which means that there is no indication of such differential NLTE depopulation of the upper level.

The plot $\log F \lambda^{3} / g f$ vs. $E P_{\mathrm{u}}$ (upper level excitation potential) of the forbidden lines, which is very similar to the figure in Viotti et al. (Viotti et al. 2001), gives evidence of good linearity for all reliable forbidden line measurements. We can conclude that the populations of these 


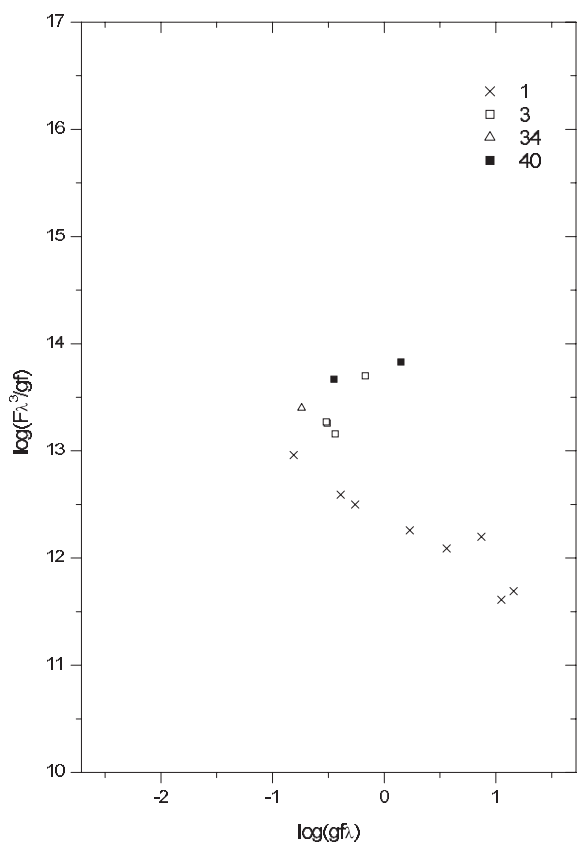

(a)

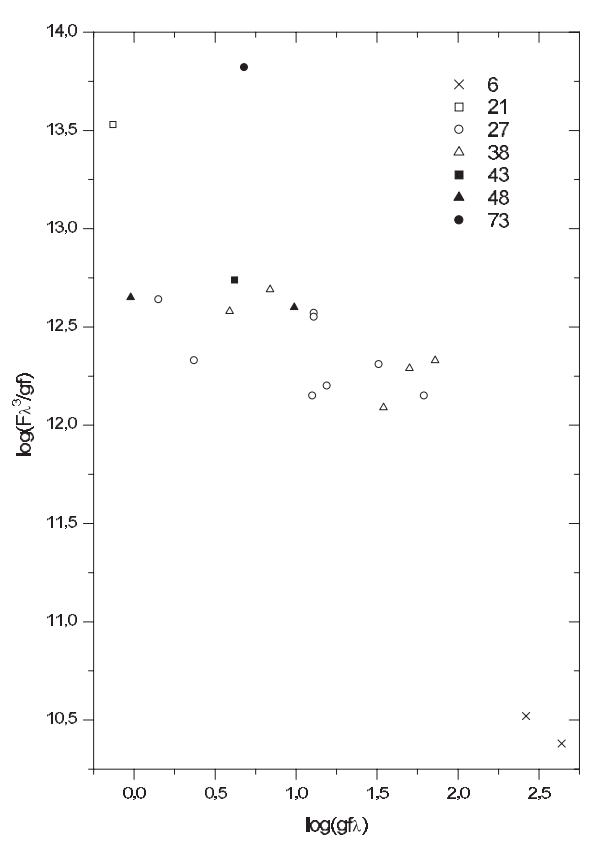

(b)

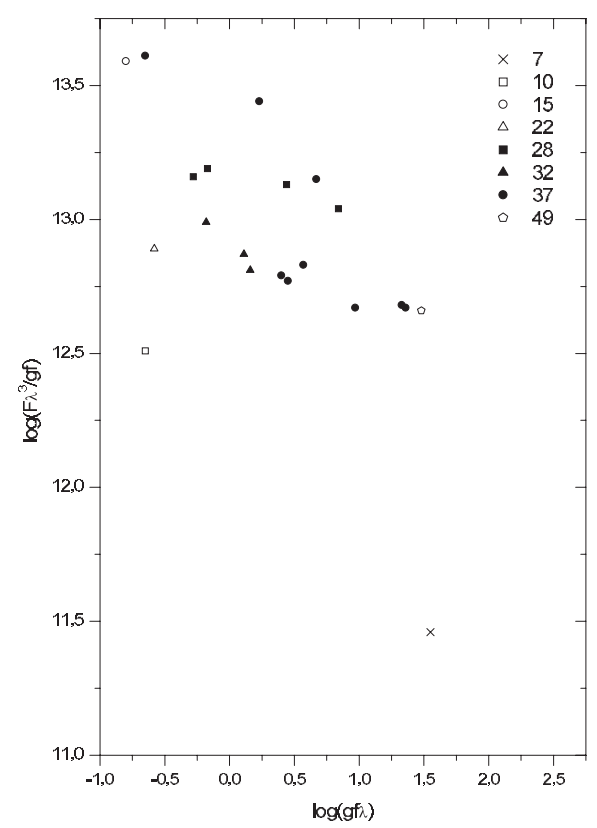

(c)

Fig. 3. Self absorption curves of multiplets with a common upper term a) $\mathrm{z}^{6} \mathrm{D}^{0}$-multiplets: $1,3,34,40$; b) $\mathrm{z}^{4} \mathrm{D}^{0}-$ multiplets: $6,21,27,38,43,48,73 ;$ c) $\mathrm{z}^{4} \mathrm{~F}^{0}$-multiplets: $7,10,15,22,28,32,37,49$.

metastable levels are quite consistent with the assumption of LTE.

On the contrary, the upper odd terms of the permitted transitions are far from linearity in the plot $\log F \lambda^{3} / g f$ vs. $E P_{\mathrm{u}}$. The scatter is partly due to self absorption, but it also suggests NLTE conditions. The NLTE effects are more pronounced for higher terms. Such lines deviate from the SAC. The deviation is larger than the scatter caused by measurement errors.

The SACs belonging to different multiplets can be used to test for NLTE effects. For example, the vertical shift between the empirical $\log$ normalized flux against $\log (g f \lambda)$ plots belonging to the multiplet pairs 27,38 and 28,37 at the same value of $\log (g f \lambda)$, is too large compared with the small difference of the mean level excitation potentials of the corresponding upper terms (less than $0.01 \mathrm{eV}$ ). This is seen in Fig. 4. Such a shift is hard to explain without invoking NLTE effects. On the other hand the apparent difference in the SAC slopes cannot be real, as there is no clear systematic effect depending on the lower term.

Among different mechanisms, NLTE pumping might be a candidate for selective excitation of certain lines, observed for highly excited upper levels of the UV lines of RR Tel (Hartmann \& Johansson 2000). However, we do not believe that this effect influences much our less excited lines.

\subsection{Test on reddening}

Apart from the already corrected interstellar reddening (Crawford et al. 1999), the expected circumstellar

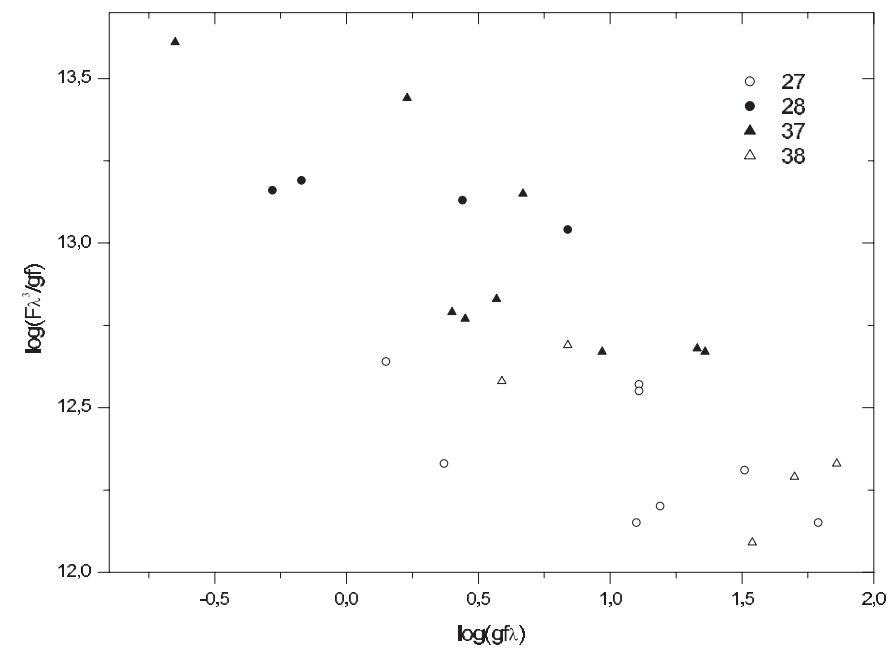

Fig. 4. Comparison of SACs belonging to the multiplet pair 27,38 with common upper term $\mathrm{z}^{4} \mathrm{D}^{0}$ (open symbols) with those of the multiplet pair 28, 37 with common upper term $\mathrm{z}^{4} \mathrm{~F}^{0}$ (filled symbols).

absorption could have an influence on our results. The SACs belonging to particular multiplets offer the possibility to test for this effect.

The comparison of the log normalized flux against $\log (g f \lambda)$ plots belonging to multiplets 1 and 40 with a common upper term $\mathrm{z}^{6} \mathrm{D}^{0}$ (Fig. 3a) suggests that the effect of reddening is small. The lines of multiplet 40 should not have a very large optical thickness, as their lower level excitation potentials are $0.20 \mathrm{eV}$ larger than the mean for the lower levels of multiplets 27 and 28 and $0.06 \mathrm{eV}$ larger 
than that for multiplets 37 and 38, while the SACs of those multiplets appear to have only moderate slopes and could be bending in the same $\log (g f \lambda)$ range (see Fig. 4). The positions of the two points of the multiplet $40 \mathrm{SAC}$ graph also suggest by themselves that the optical thickness of that multiplet is not large. If circumstellar reddening was important, the values of $\log \left(F \lambda^{3} / g f\right)$ of the lines of multiplet 40 , seen at $6433 \AA$ and $6369 \AA$, would have to be considerably larger than the corresponding values for the optically thicker lines of multiplet 1 . Multiplet 1 has a lower level excitation potential $1.84 \mathrm{eV}$ below that of multiplet 40, wavelengths of the order of $3200 \AA$ and a SAC slope of -0.7 . The observed purely horizontal shift between multiplets 1 and 40 would then be hard to understand in the presence of much reddening.

Another test exists in the comparison of multiplets with the same upper term $\mathrm{z}^{4} \mathrm{D}^{0}$ (Fig. 3b). The comparison of $\log \left(F \lambda^{3} / g f\right)$ of the observed multiplet 73 line at $7711 \AA$ with the corresponding values for multiplets 27 and 38 at low $\log (g f \lambda)$ might indicate a larger effect of dust than the comparison of multiplets 1 and 40. However, the measured multiplet 21 line, with the same upper term but a much smaller wavelength of $4177 \AA$ than the multiplet 73 line, also has a similar exceptionally large normalized flux of the order of 1 dex above the values of the lines with a similar $\log (g f \lambda)$ of other multiplets having that upper term. This suggests that reddening is not the effect which dominates. Moreover in the case of the $4177 \AA$ line, the lower level excitation potential is close to that of the multiplet 27 lines. Non-reddening effects could include for instance errors in the oscillator strengths, an unidentified blend of the $7711 \AA$ multiplet 73 line, etc.

Let us note that the log normalized flux against $\log (g f \lambda)$ plot of multiplet 73 of the Be star $\chi$ Oph is about 0.3 dex above the plot for other multiplets (Chauville et al. 2000). That star does not have dust absorption, and the effect there is probably due to the smaller optical thickness of multiplet 73 .

This leads us to the conclusion that the observed Fe II emission presumably comes from outside the region of dust which surrounds the cool component, so that reddening should not have a major effect on our results. Such a conclusion suggests that, as one might expect, iron is not singly ionized in regions where ionizing radiation is strongly absorbed by dust.

\subsection{Radius determination}

It is likely, the Fe II emission lines of RR Tel have origin in an inhomogeneous region.

In the present work we do not assume a particular form for $Q\left(\tau_{0}\right)$ when determining population ratios, it being difficult to predict theoretically or determine exactly observationally in view of the scatter of points on the log normalized flux $\log (g f \lambda)$ graphs. In the radius calculations, the $F W H M$ for line broadening along the line of sight is taken to be $14 \mathrm{~km} \mathrm{~s}^{-1}$ and the distance of our object as 3600 pc (Feast et al. 1983; Hayes \& Nussbaumer 1986).
The radius and population ratio errors appear to be dominated by the scatter of points in the log normalized flux $\log (g f \lambda)$ graphs, this being perhaps due to weak blends. Relative flux errors produce population ratio errors of generally not much more than 0.1 dex; the corresponding log radius error, taking into account absolute flux errors, is not larger.

\section{Shifts of the empirical log normalized flux against $\log (g f \lambda)$ plots}

A limit to the metastable level excitation temperature is found from multiplets 1 and 40 with a common upper term, the former being optically thick. The latter should, as already discussed, be optically thinner than multiplet 1 . The minimum horizontal shift between multiplets 1 and 40 obtained in this way, leads to an upper limit to the metastable level excitation temperature $T_{\max }=6600 \mathrm{~K}$, which in general will not be equal to the Eq. (3) temperature corresponding to the ratio of the upper to lower level populations of multiplets 1 and 40. The metastable level temperature obtained from such a horizontal shift should in general be larger than the Eq. (3) temperature, because the upper level can be expected to be depopulated with respect to the metastable level in diffuse envelopes. We can conclude that the $T$ of Eq. (3) should in any case be lower than the upper limit obtained from the horizontal shift between multiplets 1 and 40. This temperature, in agreement with Eq. (4), leads to a lower limit of the radius $R_{\text {min }}$ of the line emitting region of multiplet 40 . The assumption $\tau_{0}=1$ for the stronger line of multiplet 40 with $\lambda=6433 \AA$ would give $R_{\min }=1.8 \times 10^{12} \mathrm{~cm}$.

The assumption $\tau_{0}=1$ is clearly dubious and leads to uncertainties in the calculated $R_{\min } . R_{\min }$ is however not very sensitive to changes of $\tau_{0}$ from 1 to infinity. When $\tau_{0} \rightarrow \infty$ one obtains a slightly smaller value for $R_{\min }$ $\left(1.4 \times 10^{12} \mathrm{~cm}\right)$ than when $\tau_{0}=1$. On the contrary, $R_{\min }$ increases very fast with decreasing optical thickness towards zero, which means that supposing $\tau_{0}$ in the interval $0<\tau_{0}<1$ would affect our result very much. In that case we would determine a larger radius than that we have now determined; our radius determination would therefore still be a minimum.

\section{Common metastable level}

Making the assumption that the observed forbidden lines come from the same region as the permitted lines, we have determined the permitted line upper level/metastable level population ratio temperature by noting that the upper metastable term of forbidden lines is the same as the lower term of certain permitted lines. In our case these terms are: $b^{4} \mathrm{P}, \mathrm{b}^{4} \mathrm{~F}, \mathrm{a}^{6} \mathrm{~S}$ and $\mathrm{a}^{4} \mathrm{G}$, being common to the multiplets: (permitted 27, 28 and forbidden $4 \mathrm{~F}, 18 \mathrm{~F}$ ), (permitted $37,38,39$ and forbidden $6 \mathrm{~F}$, 20F), (permitted 40, 41, 42, 43 and forbidden $7 \mathrm{~F}$ ) and (permitted 48, 49 and forbidden $21 \mathrm{~F}$ ) respectively.

If most forbidden line emission comes from another region, the underestimated Eq. (3) temperatures in that region lead to the Eq. (4) radii which are upper limits. 
The major problem of this method is the definition of the SAC plateau of certain multiplets and the position of $\tau=1$. This is clear from the Fig. 3b showing multiplets with the same upper term, by comparing the one line of multiplet 73 , whose flux was measured, with multiplets 27 and 38. The value of the multiplet $73 \operatorname{line} \log \left(F \lambda^{3} / g f\right)$ is much larger than one might expect from the SACs of multiplets 27 and 38 according to the simple uniform slab SAC for any possible value of the optical thickness. If this effect were real, this could mean that the real SAC shape, the line optical thicknesses and the expected $\log (g f \lambda)$ of a line of a particular multiplet with $\tau_{0}=1$ could be extremely uncertain. In fact the use of the normalized fluxes of permitted lines of multiplets 27 and 38 really having $\tau_{0}>1$ would in that situation, when their optical thicknesses were assumed to be near 1, give an additional reason for the permitted upper/lower level population ratio, obtained from the comparison of permitted line and forbidden line normalized fluxes, to be a lower limit. The derived Eq. (3) temperature would of course still be as before a lower limit. On the other hand, as already mentioned, the larger value of $\log \left(F \lambda^{3} / g f\right)$ of the observed multiplet 73 line might possibly be explained by uncorrected circumstellar reddening. In that case the choice of its normalized flux to define the SAC plateau of multiplets 27 and 38 would clearly not be justified.

We use for our determinations the permitted multiplet 27 , for which quite many lines have been measured and whose empirical $\log$ normalized flux $\log (g f \lambda)$ graph shows a fairly small dispersion. Making a rather conservative assumption, $\tau_{0}$ is at least equal to 1 for $\log (g f \lambda)=1$ (with the wavelength expressed in $\AA$ ) (Figs. 2a, 3b). We deduce maximum radii for line emission of multiplet 27 making two assumptions. Firstly we suppose that $\tau_{0}$ of multiplet 27 is equal to 1 at $\log (g f \lambda)=1$ with a $\log$ normalized flux equal to 12.3 (expressing the wavelengths in Aand the line fluxes in units of $10^{-12} \mathrm{erg} \mathrm{cm}^{-2} \mathrm{~s}^{-1}$ ). If we compare the $\log$ normalized flux of the SAC plateau obtained by a correction equal to +0.2 for the self absorption at $\tau=1$ with the log normalized flux of the optically thin lines of multiplet $4 \mathrm{~F}$ of 18.4 (Fig. 5), the log population ratio of 5.9 corresponds to a temperature lower limit of $2500 \mathrm{~K}$ and a maximum radius $R_{\max }=1.6 \times 10^{14} \mathrm{~cm}$. Alternatively we can assume that the normalized flux of multiplet 73 defines the plateau of the SAC of multiplet 27 at a $\log$ normalized flux of 13.85 , because multiplet 27 has the same upper term. Using the flux and upper level excitation potential of the line at $4129 \AA$ of multiplet 27 with the lowest $g f \lambda$, which must with this assumption still be optically thick, we obtain a temperature lower limit of $3300 \mathrm{~K}$ and $R_{\max }=1.5 \times 10^{13} \mathrm{~cm}$. Of these two radius values the first is certainly an upper limit, while the other lower value is much less trustworthy.

The same procedure applied to the permitted line of multiplet 40 with $\lambda=6432.65 \AA$ and $F=0.056 \times$ $10^{-12} \mathrm{erg} \mathrm{cm}^{-2} \mathrm{~s}^{-1}$, assuming $\tau_{0}=1$ and taking for its lower level the upper term of the forbidden multiplet $7 \mathrm{~F}$, gives $T_{\min }=2300 \mathrm{~K}$ and $R_{\max }=4.9 \times 10^{13} \mathrm{~cm}$. The

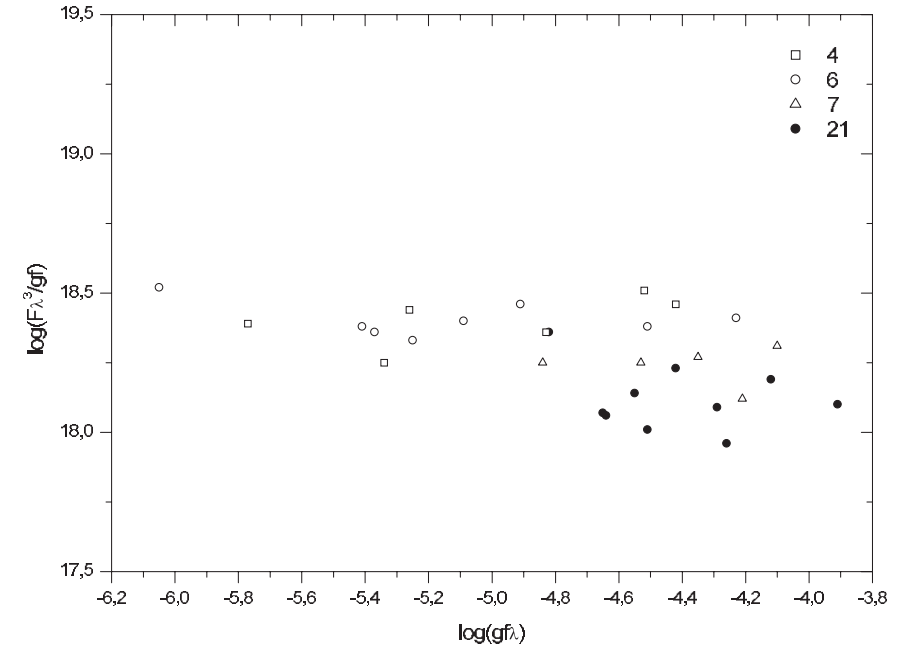

Fig. 5. Self absorption curves for the [Fe II] lines.

approximate estimate of $R_{\max }$, as already mentioned, depends on the uncertain optical thickness of the line.

\section{Forbidden line intensity condition assuming} Boltzmann distribution of metastable levels

The plot $\log F \lambda^{3} / g f$ versus upper level excitation potential $E P_{\mathrm{u}}$ for all forbidden lines assumed to be optically thin, suggests a linear dependence with a slope of about -0.76 . This leads to a temperature $T=6600 \mathrm{~K}$, which is approximately equal to the maximum permitted line region excitation temperature determined using multiplets 1 and 40. A very similar temperature with an error of $350 \mathrm{~K}$, obtained using slightly different values for $g f$, has been found in the study by Viotti et al. (2001).

We obtain the radius of the formation region of the forbidden lines from the forbidden line intensity condition. The SACs of the stronger forbidden lines, whose flux measurements are the most certain, are horizontal (Fig. 5), so we can deduce that their optical thicknesses are not more than 1. In particular the flux of the line $4244 \AA$ with the largest $\log (g f \lambda)$ of multiplet $21 \mathrm{~F}$, having such a SAC, is $4.8 \times 10^{-13} \mathrm{erg} \mathrm{cm}^{-2} \mathrm{~s}^{-1}$, so assuming a maximum thickness of 1 and a $T$ of $6600 \mathrm{~K}$,

$R_{\min }=5.3 \times 10^{12} \mathrm{~cm}$.

The same procedure applied to the line $4416 \AA$ of multiplet $6 \mathrm{~F}$ gives

$R_{\min }=4.8 \times 10^{12} \mathrm{~cm}$.

Let us emphasize that the optical thickness is probably much less than 1 , so that the actual radius is considerably larger.

The question as to whether the permitted and forbidden lines are formed in the same region can be tested observationally. The different permitted and forbidden line widths suggest that this is not quite so. The forbidden lines are slightly wider, which is clear from Fig. 6. The forbidden lines are most probably formed further out, in an accelerated wind, or more precisely in that part of the wind which is ionized by the hot component. Let it be 


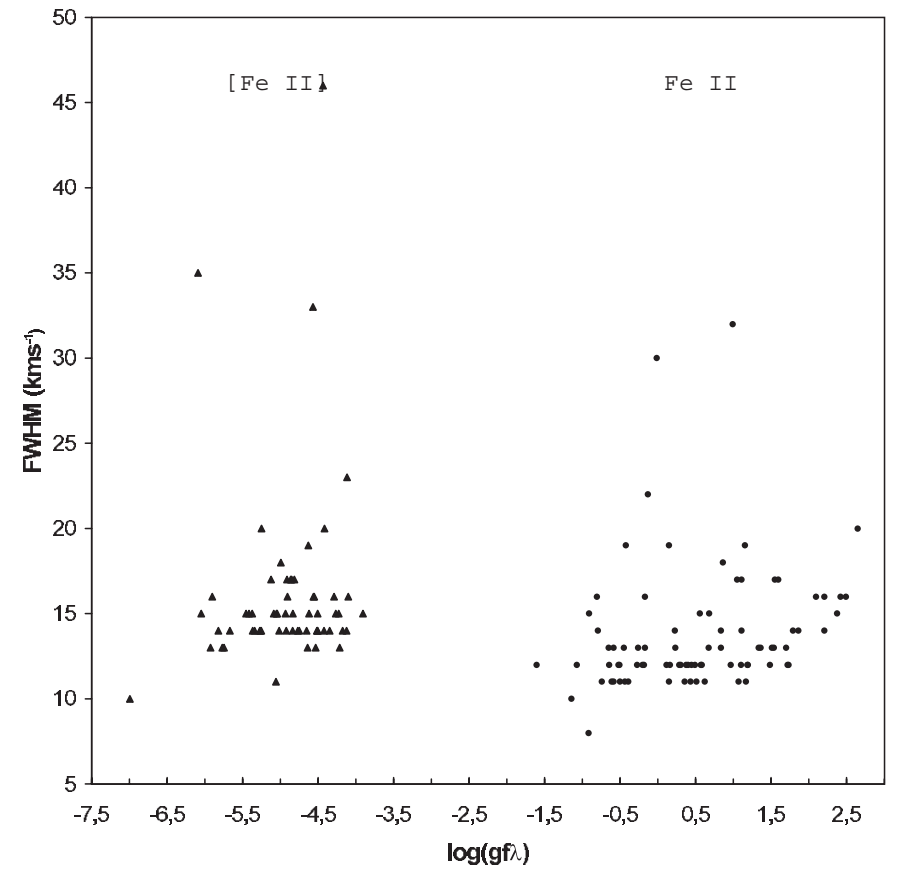

Fig. 6. Evidence for a different permitted and forbidden line forming region.

noted that both types of line have, however, the same line centre radial velocity.

The limits to temperature and radius of the Fe II region together with the procedure used to obtain them are given in Table 3.

\subsection{Column density and cool component mass loss rate}

The minumum multiplet $27 \tau_{0}$ of 1 at $\log (g f \lambda)=$ 1 leads from Eq. (2) to a minimum $N_{\mathrm{l}} / g_{\mathrm{l}}$ at $2.68 \mathrm{eV}$ of $5.3 \times 10^{14} \mathrm{~cm}^{-2}$. The maximum metastable level excitation temperature of $6600 \mathrm{~K}$, taking account of an interpolated value of the partition function of antilog 1.71 at this temperature, yields a minimum column density of $3.0 \times 10^{18}$ atoms $\mathrm{cm}^{-2}$ for $\mathrm{Fe}^{+}$and $\mathrm{Fe}$. This gives $7.5 \times 10^{22}$ atoms of hydrogen per $\mathrm{cm}^{2}$, assuming standard abundances. These results are shown in Table 4 .

Instead of our minimum radii, it is more realistic to take the large radius of the cool component, when estimating the mass loss rate. Using bolometric corrections to the $K$ magnitude for $M$ giants (Houdashelt et al. 2000), measured properties of "group 1" Mira variables with a 387 day pulsation period (Barthès et al. 1999) and an M7 spectral class of the cool component (Mürset \& Schmid 1999), we can expect a mean cool component radius of the order of $1.5 \times 10^{13} \mathrm{~cm}$ to $3.0 \times 10^{13} \mathrm{~cm}$. A slightly larger bolometric correction, obtained using the expressions of Whitelock et al. (2000), has no significant influence on this result. The smaller of these radii is not more than our maximum radii. In fact we expect line formation outside the radius of dust condensation, which according to the anonymous referee, should be at 5 Mira radii or averageing $1.0 \times 10^{14} \mathrm{~cm}$, which only seriously disagrees with our less trustworthy upper limit, found using the multiplet 73 normalized flux. A radius of 5 Mira radii leads to a minimum mass loss rate of $3.5 \times 10^{-6} M_{\mathrm{o}} \mathrm{yr}^{-1}$.

Mass loss rates for normal Miras with a similar pulsation period were determined by Loup et al. (1993) from millimeter observations of $\mathrm{CO}$ emission. The value given for the carbon rich Mira R For is $2.2 \times 10^{-6} M_{\mathrm{o}} \mathrm{yr}^{-1}$, while for the oxygen rich Miras R LMi, R Hya and S Vir the mass loss rates given are 2.8, 1.4 and $4.1 \times 10^{-7} M_{\mathrm{o}} \mathrm{yr}^{-1}$ respectively. It must be noted however that the cool components of symbiotic binaries have according to Seaquist \& Taylor (1990) larger mass loss rates than single cool giants of the same spectral type; this is to be expected if, as is believed, symbiotic activity depends on the rate of capture of the wind by the hot companion. Cool components in binaries with a suitable hot white dwarf companion, having a larger mass loss rate, would then be more likely to be considered symbiotic.

Mass loss rates have also been determined by other authors for RR Tel itself, using various methods. Seaquist et al. (1993) find a mass loss rate of $5.3 \times 10^{-6} M_{\mathrm{o}} \mathrm{yr}^{-1}$ from the flux of thermal radio emission. They used an expression for the ionization of the wind of a single hot star, having a velocity of $30 \mathrm{~km} \mathrm{~s}^{-1}$; the wind of a binary will be less ionized, the correction being larger for a larger mass loss rate, but the correction for the lower velocity of the wind of RR Tel will tend to cancel that correction. Kenyon et al. (1988) used infrared flux ratios derived from IRAS satellite observations to obtain from the infrared emission a mass loss rate of $8 \times 10^{-6} M_{\mathrm{o}} \mathrm{yr}^{-1}$. Whitelock et al. (1994) found a mass loss rate of $2.5 \times 10^{-6} M_{\mathrm{o}} \mathrm{yr}^{-1}$ from a mean relation for Miras involving their luminosities. Finally Birriel et al. (2000) found a mass loss rate from the proportion of O VI resonance line emission which is Raman scattered in parts of the wind where hydrogen is neutral. They obtain a mass loss rate of only $1 \times 10^{-6} M_{\mathrm{o}} \mathrm{yr}^{-1}$. One may wonder whether the low proportion of scattered photons and the derived lower mass loss rate are due to dust absorption of some of the far ultraviolet O VI photons. Dust absorption should be large in that wavelength region.

\section{Conclusions}

In our present approach we have tried to make as few assumptions as possible about the shape of the self absorption curve. Our results support the idea that both the permitted Fe II and the forbidden Fe [II] lines are formed in the wind of the cool Mira component, above the regions where absorption by dust is important, except perhaps in the far ultraviolet. No non-LTE effect was found between the populations of levels in the same upper term of permitted lines. However a systematic difference was found between the populations of two upper terms with a very 
Table 3. Limits on temperature and radius of the emitting region.

\begin{tabular}{|c|c|c|c|c|c|}
\hline \multicolumn{2}{|c|}{$T(K)$} & \multicolumn{2}{|c|}{$R(\mathrm{~cm})$} & \multirow[b]{2}{*}{$S A C$} & \multirow[b]{2}{*}{ procedure } \\
\hline min & $\max$ & min & $\max$ & & \\
\hline & 6600 & $1.810^{12}$ & & $\begin{array}{l}\text { multiplets } 1 \text { and } 40 \\
\text { line } 6433 \mathrm{~A}(40)\end{array}$ & $\begin{array}{l}\text { horizontal } \\
\text { shift }\end{array}$ \\
\hline 2500 & & & $1.610^{14}$ & $\begin{array}{l}\text { virtual line } 4280 \mathrm{~A}(27),=1, \log (\mathrm{gf})=1, \tilde{\mathbf{f}}=12.3 \\
\text { multiplet } 4 \mathrm{~F}, \boldsymbol{f}=18.4\end{array}$ & \multirow{3}{*}{$\begin{array}{c}\text { common } \\
\text { metastable } \\
\text { level }\end{array}$} \\
\hline 3300 & & & $1.510^{13}$ & $\begin{array}{l}\text { line } 4129 \mathrm{~A}(27), \quad, \text { plateau }(27)=\mathbf{f}(73)=13.85 \\
\text { multiplet } 4 \mathrm{~F}, \tilde{\mathbf{f}}=18.4\end{array}$ & \\
\hline \multirow[t]{4}{*}{2300} & & & $4.910^{13}$ & $\begin{array}{l}\text { line } 6433 \mathrm{~A}(40)=1 \\
\text { multiplet } 7 \mathrm{~F}, \mathbf{f}=18.2\end{array}$ & \\
\hline & 6600 & & & Boltzmann distribution of metastable levels & \multirow{3}{*}{$\begin{array}{l}\text { forbidden } \\
\text { lines }\end{array}$} \\
\hline & & $5.3 \quad 10^{12}$ & & line $4244 \mathrm{~A}(21 \mathrm{~F}) \quad \log (\mathrm{gf})=\max$ & \\
\hline & & $4.810^{12}$ & & line $4416 \mathrm{~A}(6 \mathrm{~F}) \quad \log (\mathrm{gf})=\max$ & \\
\hline
\end{tabular}

Note: $\mathbf{f}=\log \left(\mathrm{F}^{3} / \mathrm{gf}\right)$

Table 4. Minimum column density of $\mathrm{Fe}$ and $\mathrm{H}$ and the cool component mass loss rate.

\begin{tabular}{|c|c|c|c|c|c|c|c|c|c|c|c|}
\hline \multirow{3}{*}{$\mathrm{T}(\mathrm{K})$} & \multirow{2}{*}{\multicolumn{2}{|c|}{$\begin{array}{l}\text { Minimum column density } \\
\qquad\left(\mathrm{cm}^{-2}\right)\end{array}$}} & \multirow{2}{*}{\multicolumn{2}{|c|}{$\mathrm{R}(\mathrm{cm})$}} & \multicolumn{7}{|c|}{ Mass loss rate $\left(\right.$ Ho $\left.\mathrm{yr}^{-1}\right)$} \\
\hline & & & & & \multicolumn{5}{|c|}{ RR Tel } & \multicolumn{2}{|c|}{$\begin{array}{c}\text { normal Miras } \\
\text { Loup et al. (1993) }\end{array}$} \\
\hline & $\mathrm{Fe}$ & $\mathrm{H}$ & $\begin{array}{l}\text { dust formation } \\
\text { region } \\
\text { (referee) }\end{array}$ & $\begin{array}{c}\mathrm{R}_{\mathrm{c}} \\
\text { cool Mira } \\
\text { component }\end{array}$ & $\begin{array}{c}\boldsymbol{M}_{\min } \\
\text { (this paper) }\end{array}$ & $\begin{array}{l}\text { Whitelock et } \\
\text { al. (1994) }\end{array}$ & $\begin{array}{c}\text { Seaquist et al. } \\
\text { (1993) }\end{array}$ & $\begin{array}{l}\text { Kenyon et } \\
\text { al. (1988) }\end{array}$ & $\begin{array}{l}\text { Birriel et al. } \\
(2000)\end{array}$ & $\begin{array}{l}\text { carbon } \\
\text { rich }\end{array}$ & $\begin{array}{l}\text { oxygen } \\
\text { rich }\end{array}$ \\
\hline 6600 & $3.0 \cdot 10^{18}$ & $7.5 \cdot 10^{22}$ & $\begin{array}{c}5 R_{C} \\
\left(1.0 \cdot 10^{14}\right)\end{array}$ & $\begin{array}{l}1.5 \cdot 10^{13} \\
3.0 \cdot 10^{13}\end{array}$ & $3.5 \cdot 10^{-6}$ & $2.5 \cdot 10^{-6}$ & $5.3 \cdot 10^{-6}$ & $8.0 \cdot 10^{-6}$ & $1.0 \cdot 10^{-6}$ & $2.2 \cdot 10^{-6}$ & $\begin{array}{l}2.8 \cdot 10^{-7} \\
1.4 \cdot 10^{-7} \\
4.1 \cdot 10^{-7}\end{array}$ \\
\hline
\end{tabular}

Note: $\boldsymbol{M}_{\mathrm{min}}$ is the mass loss rate calculated by use of the minimum column density and the radius of the dust formation region

similar upper term excitation potential. The SAC method offers many possibilities for this kind of test.

We note that the SAC method is best applied to lines in the optical region. The populations of the upper levels of many ultraviolet lines appear to have anomalous populations due to selective pumping mechanisms (Hartmann \& Johansson 2000).

Acknowledgements. We wish to thank Marie-Odile Mennessier and Patricia Whitelock for useful information about mira variables, Francis Keenan for information about the fluxes tabulated in Crawford et al. (1999) and the anonymous referee. Thanks are due also to R. Viotti for instructive discussions on atomic parameters and to Gerard Muratorio for supplying some gf values. Corinne Rossi, who read a preliminary version of the paper, gave useful advice. D. Kotnik-Karuza acknowledges the kind hospitality of the Institut d'Astrophysique, Paris. This visit was supported by a grant from the French government.

\section{References}

Baratta, G. B., Friedjung, M., Muratorio, G., Rossi, C., \& Viotti, R. 1998, The Self Absorption Curve Method, A Users' Manual, IAS Int. Rep., December 1998

Barthès, D., Luri, X., Alvarez, R., \& Mennessier, M. O. 1999, A\&AS, 140,55

Birriel, J. J., Espey, B. R., \& Schulte-Ladbeck, R. E. 2000, ApJ, 545, 1020

Chauville, J., Ballereau, D., Cidale, L., et al. 2000, in The Be Phenomenon in Early-Type Stars, ed. M. A. Smith, H. F. Henrichs, \& J. Fabregat (Astronomical Society of the Pacific), 523

Contini, M., \& Formiggini, L. 1999, ApJ, 517, 925

Crawford, F. L., McKenna, F. C., Keenan, F. P., et al. 1999, A\&AS, 139, 135

Feast, M. W., \& Glass, I. S. 1974, MNRAS, 167, 81

Feast, M. W., Whitelock, P. A., Catchpole, R. M., Roberts, G., \& Carter, B. S. 1983, MNRAS, 202, 951

Friedjung, M., \& Muratorio, G. 1980, A\&A, 85, 233

Friedjung, M., \& Muratorio, G. 1987, A\&A, 188, 100 
Garstang, R. H. 1962, MNRAS, 124, 321

Hansen, J. E., \& Lister, G. M. S. 1988, in Physics of Formation of FeII Lines Outside LTE, ed. R. Viotti, A. Vittone, \& M. Friedjung (D. Reidel Publishing Company), 51

Hartmann, H., \& Johansson, S. 2000, A\&A, 359, 627

Hayes, M. A., \& Nussbaumer, H. 1986, A\&A, 161, 287

Houdashelt, M. L., Bell, R. A., Sweigart, A. V., \& Wing, R. F. 2000, AJ, 119, 1424

Kastner, S. O. 1999, A\&A, 351, 1016

Keenan, F. P., private communication

Kenyon, S. J., Fernandez-Castro, T., \& Stencel, R. E. 1988, AJ, 95, 1817

Kurucz, R. L. 1981, Smithsonian Astrophys. Obs. Special Report No. 390 and CD Rom data [UVSPACE.LINES]FeIIKURUCZ.LIN

Loup, C., Forveille, T., Omont, A., \& Paul, J. F. 1993, A\&AS, 99, 291

McKenna, F. C., Keenan, F. P., Hambly, N. C., et al. 1997, ApJS, 109, 225

Moore, Ch. E. 1945, A Multiplet Table of Astrophysical Interest (Princeton, N.J.)

Mürset, U., \& Schmid, H. M. 1999, A\&AS, 137, 473

Muratorio, G. 1999, private communication
Muratorio, G., \& Friedjung, M. 1988, A\&A, 190, 103

Muratorio, G., Viotti, R., Friedjung, M., Baratta, G. B., \& Rossi, C. 1992, A\&A, 258, 423

Nussbaumer, H., Pettini, M., \& Storey, P. J. 1981, A\&A, 102, 351

Nussbaumer, H., \& Storey, P. J. 1988, A\&A, 193, 327

Penston, M. V., Benvenuti, P., Cassatella, A., et al. 1983 MNRAS, 202, 833

Purton, C. R., Feldman, P. A., Marsh, K. A., Allen, D. A., \& Wright, A. E. 1982, MNRAS, 198, 321

Seaquist, E. R., \& Taylor, A. R. 1990, ApJ, 349, 313

Seaquist, E. R., Krogulec, M., \& Taylor, A. R. 1993, ApJ, 410, 260

Selvelli, P. L., \& Bonifacio, P. C. 2000, A\&A, 364, L15

Thackeray, A. D. 1977, Mem. R. astr. Soc. 83, 1

Verner, E. M., Verner, D. A., Korista, K. T., et al. 1999, ApJS, 120, 101

Viotti, R. F., Baratta, G. B., Friedjung, M., Rossi, C., \& Spector, N. 2001, A\&A, submitted

Whitelock, P., Menzies, J., Feast, M., et al. 1994, MNRAS, 267, 711

Whitelock, P., Marang, F., \& Feast, M. 2000, MNRAS, 319, 728 